\title{
G-Strands and Peakon Collisions on $\operatorname{Diff}(\mathbb{R})^{\star}$
}

\author{
Darryl D. HOLM ${ }^{\dagger}$ and Rossen I. IVANOV $\ddagger$ \\ † Department of Mathematics, Imperial College London, London SW7 2AZ, UK \\ E-mail:d.holm@ic.ac.uk \\ URL: http://www2.imperial.ac.uk/ dholm/ \\ ¥ School of Mathematical Sciences, Dublin Institute of Technology, \\ Kevin Street, Dublin 8, Ireland \\ E-mail: rivanov@dit.ie
}

Received October 29, 2012, in final form March 21, 2013; Published online March 26, 2013

http://dx.doi.org/10.3842/SIGMA.2013.027

\begin{abstract}
A $G$-strand is a map $g: \mathbb{R} \times \mathbb{R} \rightarrow G$ for a Lie group $G$ that follows from Hamilton's principle for a certain class of $G$-invariant Lagrangians. Some $G$-strands on finite-dimensional groups satisfy $1+1$ space-time evolutionary equations that admit soliton solutions as completely integrable Hamiltonian systems. For example, the $\mathrm{SO}(3)$-strand equations may be regarded physically as integrable dynamics for solitons on a continuous spin chain. Previous work has shown that $G$-strands for diffeomorphisms on the real line possess solutions with singular support (e.g. peakons). This paper studies collisions of such singular solutions of $G$-strands when $G=\operatorname{Diff}(\mathbb{R})$ is the group of diffeomorphisms of the real line $\mathbb{R}$, for which the group product is composition of smooth invertible functions. In the case of peakon-antipeakon collisions, the solution reduces to solving either Laplace's equation or the wave equation (depending on a sign in the Lagrangian) and is written in terms of their solutions. We also consider the complexified systems of $G$-strand equations for $G=\operatorname{Diff}(\mathbb{R})$ corresponding to a harmonic map $g: \mathbb{C} \rightarrow \operatorname{Diff}(\mathbb{R})$ and find explicit expressions for its peakon-antipeakon solutions, as well.
\end{abstract}

Key words: Hamilton's principle; continuum spin chains; Euler-Poincaré equations; Sobolev norms; singular momentum maps; diffeomorphisms; harmonic maps

2010 Mathematics Subject Classification: 37J15; 37K05; 35R01

In honor of Peter Olver's sixtieth birthday. Happy birthday, Peter!

\section{Euler-Poincaré equations for a $G$-strand}

The Euler-Poincaré (EP) theory of $G$-strands is an extension of the classical chiral models to include norms that are not bi-invariant $[13,14]$. The classical chiral models are nonlinear relativistically invariant Lagrangian field theories on group manifolds. As such, they are fundamental in theoretical physics. The vast literature of results for these models is fascinating. For example, it is well known that these models are integrable in $1+1$ dimensions and possess soliton solutions. See $[2,3,19,20,22,23,31,32]$ and references therein for discussions of the many aspects of integrability of the chiral models, including the famous dressing method for explicitly deriving the soliton solutions of these models, which is given in [31]. The solitons for the $\mathrm{O}(3)$ chiral model are particularly familiar [30], because this model allows an integrable reduction to the one-component sine-Gordon equation (e.g. see [22]). Many generalizations of these models have been introduced. For example, generalized chiral models with metrics that are not ad-invariant on the Lie algebra are considered in [25]. Other generalizations of chiral models

\footnotetext{
${ }^{\star}$ This paper is a contribution to the Special Issue "Symmetries of Differential Equations: Frames, Invariants and Applications". The full collection is available at http://www.emis.de/journals/SIGMA/SDE2012.html
} 
for non-semisimple groups are studied in [11]. An integrable chiral model in $2+1$ dimensions was proposed in [28] and the possibility for fermionic interpretation of the current variables was explored in [29]. Finally, their formulation as Euler-Poincaré equations for $G$-strands with Lagrangians given as norms on tangent spaces of finite-dimensional Lie groups was accomplished in $[13,14]$.

The class of $G$-strand problems treated here generalizes the classical chiral model, which appear as a special case of the $G$-strands when their structure group is $\mathrm{SO}(3)$ and the norm defining their Lagrangian is bi-invariant, as discussed in [14]. $G$-strands are found here to also be associated with a certain generalization of harmonic maps into Lie groups. Harmonic maps into Lie groups were studied mathematically, and their relation to the chiral model via complexification of its independent coordinates was discussed in [27]. Following this same idea, we shall complexify the independent coordinates of the $G$-strand on the Lie group of diffeomorphisms $\operatorname{Diff}(\mathbb{R})$ and use the Euler-Poincaré theory to derive a class of equations which may be regarded as partial differential equations for harmonic maps of $\mathbb{C}$ into $\operatorname{Diff}(\mathbb{R})$. This particular advance is not by itself the main point of the present paper, though. The main point is the investigation of the interactions between singular solutions of the $G$-strand equations for the group of diffeomorphisms $\operatorname{Diff}(\mathbb{R})$, both as evolutionary equations and as harmonic maps.

Main content of the paper. Section 1 summarizes the derivation of the class of $G$ strand equations first studied in the context of Euler-Poincaré theory in [13, 14]. Section 2 discusses singular (peakon) solutions and their collision dynamics for the $G$-strand equations with $G=\operatorname{Diff}(\mathbb{R})$. These peakon solutions were shown to exist in [14]. Here we study the dynamics of their pairwise interactions. Section 3 introduces the complexified $\operatorname{Diff}(\mathbb{R})$-strand equations and determines their solutions corresponding to peakon collision dynamics. Section 4 summarizes the results of the paper and provides some outlook for future research.

Left $G$-invariant Lagrangian. We begin with the following ingredients of EP theory. For more details and discussion, see [13, 17].

- Let $G$ be a Lie group. The map $g:(t, s) \in \mathbb{R} \times \mathbb{R} \rightarrow g(t, s) \in G$ possesses two types of tangent vectors, $\left(u_{g}, v_{g}\right) \in T G \times T G$, corresponding to its two types of derivatives, with respect to $t$ and $s$.

- Assume that the function $L\left(g, u_{g}, v_{g}\right): T G \times T G \rightarrow \mathbb{R}$ is right $G$-invariant.

- Right $G$-invariance of $L$ permits us to define $l: \mathfrak{g} \times \mathfrak{g} \rightarrow \mathbb{R}$ by

$$
l\left(u_{g} g^{-1}, v_{g} g^{-1}\right)=L\left(g, u_{g}, v_{g}\right) .
$$

Conversely, this relation defines for any $l: \mathfrak{g} \times \mathfrak{g} \rightarrow \mathbb{R}$ a right $G$-invariant function $L$ : $T G \times T G \rightarrow \mathbb{R}$

- For a map $g(t, s): \mathbb{R} \times \mathbb{R} \rightarrow G$ one defines the right $G$-invariant tangent vectors at the identity of $G$, given by

$$
u(t, s):=g_{t} g^{-1}(t, s) \quad \text { and } \quad v(t, s):=g_{s} g^{-1}(t, s) .
$$

Lemma 1. The right-trivialized tangent vectors $u(t, s)$ and $v(t, s)$ at the identity of $G$ satisfy

$$
v_{t}-u_{s}=\operatorname{ad}_{u} v
$$

Proof. The proof is standard and follows from equality of cross derivatives $g_{t s}=g_{s t}$, cf. [13, 14, 17]. As a consequence, equation (1) is often called a zero-curvature relation.

Theorem 1 (Euler-Poincaré theorem). With the preceding notation, the following four statements are equivalent $[13,14]$ : 
(i) Hamilton's variational principle on $T G \times T G$

$$
\delta \int_{t_{1}}^{t_{2}} L\left(g(t, s), g_{t}(t, s), g_{s}(t, s)\right) d s d t=0
$$

holds, for variations $\delta g(t, s)$ of $g(t, s)$ vanishing at the endpoints in $t$ and $s$.

(ii) The function $g(t, s)$ satisfies Euler-Lagrange equations for $L$ on $G$, given by

$$
\frac{\partial L}{\partial g}-\frac{\partial}{\partial t} \frac{\partial L}{\partial g_{t}}-\frac{\partial}{\partial s} \frac{\partial L}{\partial g_{s}}=0
$$

(iii) The constrained variational principle ${ }^{1}$

$$
\delta \int_{t_{1}}^{t_{2}} l(u(t, s), v(t, s)) d s d t=0
$$

holds on $\mathfrak{g} \times \mathfrak{g}$, using variations of $u$ and $v$ of the forms

$$
\delta u=w_{t}-\operatorname{ad}_{u} w \quad \text { and } \quad \delta v=w_{s}-\operatorname{ad}_{v} w,
$$

where $w(t, s) \in \mathfrak{g}$ vanishes at the endpoints.

(iv) The Euler-Poincaré equations hold on $\mathfrak{g}^{*} \times \mathfrak{g}^{*}$

$$
\frac{d}{d t} \frac{\delta l}{\delta u}+\operatorname{ad}_{u}^{*} \frac{\delta l}{\delta u}+\frac{d}{d s} \frac{\delta l}{\delta v}+\operatorname{ad}_{v}^{*} \frac{\delta l}{\delta v}=0 .
$$

Evolutionary $G$-strand. We now define the fundamental quantity of interest in the remainder of the paper.

Definition 1. A $G$-strand is an evolutionary map into a Lie group $G, g(t, s): \mathbb{R} \times \mathbb{R} \rightarrow G$, whose dynamics in $(t, s) \in \mathbb{R} \times \mathbb{R}$ may be obtained from Hamilton's principle for a $G$-invariant reduced Lagrangian $l: \mathfrak{g} \times \mathfrak{g} \rightarrow \mathbb{R}$, where $\mathfrak{g}$ is the Lie algebra of the group $G$. The $G$-strand system of evolutionary partial differential equations for a right $G$-invariant reduced Lagrangian consists of the zero-curvature equation (1) and the Euler-Poincaré (EP) variational equations (3). ${ }^{2}$

Subclasses of the $G$-strand maps contain the principal chiral models of field theory in theoretical physics, reviewed, e.g., in [20]. An interpretation of the $G$-strand equations as the dynamics of a continuous spin chain is given in [13]. This is the source of the term, 'strand'. The corresponding theory of molecular strands is discussed in [4]. Recently, a covariant field theory of $G$-strands in higher dimensions for $G=\operatorname{Diff}(\mathbb{R})$ has also been developed [5].

Overview and organization of the paper. In Section 2, we shall first summarize $G$ strand dynamics for diffeomorphisms on the real line and recall from [14] that the Diff(R)-strand dynamics admit singular solutions associated with a pair of momentum maps. Then we shall derive the one-peakon solution and solve for the collision dynamics of two-peakon interactions on $G$-strands. In Section 3 we introduce the complexified $\operatorname{Diff}(\mathbb{R})$-strand equations and solve their partial differential equations corresponding to two-peakon collision dynamics. Thus, Section 3 investigates the interactions between singular solutions of the $G$-strand equations for the group of diffeomorphisms $\operatorname{Diff}(\mathbb{R})$, both as evolutionary equations and as harmonic maps. Finally, Section 4 summarizes the present results and provides some outlook for future research.

\footnotetext{
${ }^{1}$ As with the basic Euler-Poincaré equations [21], this is not strictly a variational principle in the same sense as the standard Hamilton's principle. It is more like the Lagrange d'Alembert principle, because we impose the stated constraints on the variations allowed [17]. The proof of equations (2) follows the same pattern as the proof of the first zero-curvature relation (1).

${ }^{2}$ The left $G$-invariant case yields similar equations, but with the opposite signs of ad and ad*, as in [14].
} 


\section{The $G$-strand PDE for $G=\operatorname{Diff}(\mathbb{R})$}

This section studies the $G$-strand system that arises when we choose $G=\operatorname{Diff}(\mathbb{R})$ and the Lagrangian involves the $H^{1}$ Sobolev norm. This case is reminiscent of fluid dynamics and may be written naturally in terms of right-invariant tangent vectors $u(t, s, x)$ and $v(t, s, x)$ defined by

$$
\partial_{t} g=u \circ g \quad \text { and } \quad \partial_{s} g=v \circ g \text {, }
$$

where the symbol $\circ$ denotes composition of functions. In this right-invariant case, the $G$-strand system of partial differential equations (PDE) with reduced Lagrangian $\ell(u, v)$ takes the following form, which generalizes to $\mathbb{R}^{d}$ in any number of spatial dimensions,

$$
\frac{\partial}{\partial t} \frac{\delta \ell}{\delta u}+\frac{\partial}{\partial s} \frac{\delta \ell}{\delta v}=-\operatorname{ad}_{u}^{*} \frac{\delta \ell}{\delta u}-\operatorname{ad}_{v}^{*} \frac{\delta \ell}{\delta v}, \quad \frac{\partial v}{\partial t}-\frac{\partial u}{\partial s}=\operatorname{ad}_{u} v .
$$

The distinction between the maps $(u, v): \mathbb{R} \times \mathbb{R} \rightarrow \mathfrak{g} \times \mathfrak{g}$ and their pointwise values $(u(t, s), v(t, s))$ $\in \mathfrak{g} \times \mathfrak{g}$ will always be clear in context, so that no confusion will arise. Likewise, for the variational derivatives $\delta \ell / \delta u$ and $\delta \ell / \delta v$.

Equations (4) form a subset of the equations studied in $[6,12,15,26]$ for complex fluids. They also form a subset of the equations for molecular strands studied in [4]. The latter comparison further justifies the name $G$-strands for the systems being studied here. These equations may be derived from Hamilton's principle for an affine Lie group action, under which the auxiliary equation for $v$ may be interpreted as an advection law. This interpretation is discussed further in $[4,6]$. The covariant form of these equations has been developed in [5].

\subsection{The $G$-strand Hamiltonian structure}

Upon setting $m=\delta \ell / \delta u$ and $n=\delta \ell / \delta v$, the right-invariant $G$-strand equations in (4) for maps

$\mathbb{R} \times \mathbb{R} \rightarrow G=\operatorname{Diff}(\mathbb{R})$ in one spatial dimension may be expressed as a system of two 1+2 PDEs in $(t, s, x)$,

$$
\begin{aligned}
& m_{t}+n_{s}=-\operatorname{ad}_{u}^{*} m-\operatorname{ad}_{v}^{*} n=-(u m)_{x}-m u_{x}-(v n)_{x}-n v_{x} \\
& v_{t}-u_{s}=-\operatorname{ad}_{v} u=-u v_{x}+v u_{x} .
\end{aligned}
$$

The corresponding Hamiltonian structure for these $\operatorname{Diff}(\mathbb{R})$-strand equations is obtained by Legendre transforming [17] to

$$
h(m, v)=\langle m, u\rangle-\ell(u, v) .
$$

One may then write the $m-v$ equations (5) in Lie-Poisson Hamiltonian form as

$$
\frac{d}{d t}\left[\begin{array}{c}
m \\
v
\end{array}\right]=\left[\begin{array}{cc}
-\operatorname{ad}_{\square}^{*} m & \partial_{s}+\operatorname{ad}_{v}^{*} \\
\partial_{s}-\operatorname{ad}_{v} & 0
\end{array}\right]\left[\begin{array}{c}
\delta h / \delta m=u \\
\delta h / \delta v=-n
\end{array}\right],
$$

where $\left(\operatorname{ad}_{\square}^{*} m\right) v:=\operatorname{ad}_{v}^{*} m$ for $v \in \mathfrak{g}$ and $m \in \mathfrak{g}^{*}$. This is the Lie-Poisson bracket dual to the action of the semidirect-product Lie algebra

$$
\mathfrak{g}=\mathfrak{X}(\mathbb{R}) \subseteq \Lambda^{1}(\text { Dens })(\mathbb{R}) \oplus C\left(\partial_{s}\right),
$$

in which $\mathfrak{X}(\mathbb{R})$ is the space of vector fields and $\Lambda^{1}$ (Dens) $(\mathbb{R})$ is the space of 1 -form densities on the real line $\mathbb{R}$, plus a generalized 2-cocycle $C\left(\partial_{s}\right)$. In the lower off-diagonal entry of the Hamiltonian matrix in (6), one recognizes the vector-field covariant derivative in $s$, and finds its negative adjoint operator in the upper off-diagonal entry. The Lie-Poisson bracket in (6) first 
arose in the Hamiltonian formulation of chromohydrodynamics, i.e., the dynamics of a YangMills fluid plasma [7, 8]. For discussions of how such Lie-Poisson Hamiltonian structures arise in complex fluids with finite-dimensional order-parameter groups (broken symmetries) and full discussions of their Lie algebraic properties, see [6, 12, 15, 26] and references therein.

Relation to the Camassa-Holm equation [1]. An interesting subcase of the system of semi-stationary $\operatorname{Diff}(\mathbb{R})$-strand equations (4) arises when one chooses the Lagrangian $\ell(u, v)$ in (4) to depend only on $u$, as its $H^{1}$ norm on the real line,

$$
\ell(u, v)=\frac{1}{2}\|u\|_{H^{1}}^{2},
$$

with vanishing boundary conditions, as $|x| \rightarrow \infty$. In this case, $m=u-u_{x x}$, and this restriction of the equations in (4) provides an extension of the completely integrable Camassa-Holm (CH) equation [1],

$$
m_{t}=-\operatorname{ad}_{u}^{*} m=-(u m)_{x}-m u_{x} \quad \text { with } \quad m=\frac{\delta \ell}{\delta u}=u-u_{x x} .
$$

Specifically, these modified $G$-strand equations reduce in the absence of $v$-dependence precisely to the $\mathrm{CH}$ equation, which admits singular solutions known as peakons in the form [1]

$$
m(x, t)=\sum_{a} M_{a}(t) \delta\left(x-Q^{a}(t)\right)
$$

where we sum in $a \in \mathbb{Z}$ over the integers, or over any subset of the integers. The peakon solution (8) of the $\mathrm{CH}$ equation may be understood as a singular momentum map obtained from the left action of $\operatorname{Diff}(\mathbb{R})$ on embeddings of points on the real line [16]. The generalization to higher dimensions is also possible. See [16] for further discussion.

\subsection{Peakon solutions of the $\operatorname{Diff}(\mathbb{R})$-strand equations in (5)}

With the following choice of Lagrangian using the $H^{1}$ norm,

$$
\ell(u, v)=\frac{1}{2}\|u\|_{H^{1}}^{2}+\frac{\sigma}{2}\|v\|_{H^{1}}^{2},
$$

the $\operatorname{Diff}(\mathbb{R})$-strand equations (5) admit peakon solutions in both momenta $m$ and $n$, with continuous velocities $u$ and $v$. Here $\sigma= \pm 1$ provides two possibilities for a sign choice. Note that only the choice $\sigma=-1$ leads to a positive-definite Hamiltonian. The choice $\sigma=1$ in (9) yields a conserved Hamiltonian that is not bounded below. We state the main result in the following theorem.

Theorem 2. The $\operatorname{Diff}(\mathbb{R})$-strand equations (5) admit singular solutions expressible as linear superpositions summed over $a \in \mathbb{Z}[14]$

$$
\begin{aligned}
& m(x, t, s)=\sum_{a} M_{a}(t, s) \delta\left(x-Q^{a}(t, s)\right), \\
& n(x, t, s)=\sum_{a} N_{a}(t, s) \delta\left(x-Q^{a}(t, s)\right), \\
& u(x, t, s)=K * m=\sum_{a} M_{a}(t, s) K\left(x, Q^{a}\right), \\
& v(x, t, s)=\sigma K * n=\sigma \sum_{a} N_{a}(t, s) K\left(x, Q^{a}\right),
\end{aligned}
$$


that are peakons in the case that $K(x, y)=\frac{1}{2} e^{-|x-y|}$ is the Green function of the Helmholtz operator $1-\partial_{x}^{2}$. These singular solutions follow from Hamilton's principle $\delta S=0$ for the constrained action $S=\int L(u, v, Q) d t$ given by

$$
\begin{aligned}
S= & \int \ell(u, v)+\sum_{a} M_{a}(t, s)\left(\partial_{t} Q^{a}(t, s)-u\left(Q^{a}, t, s\right)\right) \\
& +\sum_{a} N_{a}(t, s)\left(\partial_{s} Q^{a}(t, s)-v\left(Q^{a}, t, s\right)\right) d s d t .
\end{aligned}
$$

and they are peakons in $(t, s)$ for the Lagrangian $\ell(u, v)$ given in equation (9).

Proof. The proof of this theorem is a direct calculation following the method of momentum maps and the Clebsch approach for this type of problem introduced in [14]. The results for the present case are given below.

The solution parameters $\left\{Q^{a}(t, s), M_{a}(t, s), N_{a}(t, s)\right\}$ with $a \in \mathbb{Z}$ that specify the singular solutions (10) are determined by the following set of evolutionary PDEs in $s$ and $t$, in which we denote $K^{a b}:=K\left(Q^{a}, Q^{b}\right)$ with integer summation indices $b, c, e \in \mathbb{Z}$ :

$$
\begin{aligned}
& \partial_{t} Q^{a}(t, s)=u\left(Q^{a}, t, s\right)=\sum_{b} M_{b}(t, s) K^{a b}, \\
& \partial_{s} Q^{a}(t, s)=v\left(Q^{a}, t, s\right)=\sigma \sum_{b} N_{b}(t, s) K^{a b}, \\
& \left.\partial_{t} M_{a}(t, s)=-\partial_{s} N_{a}-\sum_{c}\left(M_{a} M_{c}+\sigma N_{a} N_{c}\right) \frac{\partial K^{a c}}{\partial Q^{a}} \quad \text { (no sum on } a\right), \\
& \partial_{t} N_{a}(t, s)=\sigma \partial_{s} M_{a}+\sum_{b, c, e}\left(N_{b} M_{c}-M_{b} N_{c}\right) \frac{\partial K^{e c}}{\partial Q^{e}}\left(K^{e b}-K^{c b}\right)\left(K^{-1}\right)_{a e} .
\end{aligned}
$$

The notation $\left(K^{-1}\right)_{a b}$ is for the $a b$-entry of the matrix $K^{-1}$.

The last pair of equations in (11) may be solved as a system for the momenta, or Lagrange multipliers $\left(M_{a}, N_{a}\right)$, then used in the previous pair to update the support set of positions $Q^{a}(t, s)$. Given $Q^{a}(t, s)$ for $a \in \mathbb{Z}$, one constructs $(m(x, t, s), n(x, t, s))$ along the solution paths $x=Q^{a}(t, s)$ from the first pair of (10) and then obtains $(u(x, t, s), v(x, t, s))$ for $x \in \mathbb{R}$ from the second pair. Alternatively, knowing the position $Q^{a}(t, s), a \in \mathbb{Z}$, for all $s$ at a given time $t$, also determines $N_{a}$ upon inverting the matrix $K^{a b}$ in the second equation in (11). The values of $M_{a}$ can be determined similarly.

Single-peakon solution. The single-peakon solution of the $\operatorname{Diff}(\mathbb{R})$-strand equations (5) is straightforward to obtain from (11). Namely, writing the first two equations for a single peakon and substituting into the third equation shows that $Q^{1}(t, s)$ satisfies the equation,

$$
\left(\partial_{s}^{2}+\sigma \partial_{t}^{2}\right) Q^{1}(t, s)=0
$$

Thus, any solution $h(t, s)$ of the linear equation $\left(\partial_{s}^{2}+\sigma \partial_{t}^{2}\right) h(t, s)=0$ provides a solution $Q^{1}=$ $h(t, s)$. One then finds from the first two equations in (11) that

$$
M^{1}(t, s)=\frac{1}{K_{0}} h_{t}(t, s), \quad N^{1}(t, s)=\frac{\sigma}{K_{0}} h_{s}(t, s),
$$

where $K_{0}=K(0)$ with $K(X-Y) \equiv K(X, Y)$. The solution for the single-peakon parameters $Q^{1}, M^{1}$ and $N^{1}$ depends only on the choice of the harmonic function $h(t, s)$, which in turn depends on the $(t, s)$ boundary conditions. In particular, the single-peakon does not depend on the shape of the Green's function $K(x)$. 


\subsection{Two-peakon interactions on $G$-strands}

Consider an operator norm $\|w\|_{\mathcal{Q}}^{2}=\frac{1}{2} \int w \mathcal{Q} w d x$ where the operator $\mathcal{Q}$ is symmetric, positivedefinite and spatially translation-invariant, with vanishing boundary conditions, as $|x| \rightarrow \infty$. For example, the $H^{1}$ norm for the $\mathrm{CH}$ peakons in the Lagrangian (9) arises when $\mathcal{Q}$ is chosen to be the Helmholtz operator, so that $\mathcal{Q} w=w-w_{x x}$.

Let the Lagrangian be

$$
\ell(u, v)=\frac{1}{2}\|u\|_{\mathcal{Q}}^{2}+\frac{\sigma}{2}\|v\|_{\mathcal{Q}}^{2} .
$$

Consider peakons at positions $Q^{1}(t, s)$ and $Q^{2}(t, s)$ on the real line. The Green's function $K$ for the operator $\mathcal{Q}$ depends only on the difference of the peakon positions, $X(t, s)=Q^{1}-Q^{2}$. Then the first two equations in (11) imply

$$
\partial_{t} X=\left(M_{1}-M_{2}\right)\left(K_{0}-K(X)\right), \quad \partial_{s} X=\sigma\left(N_{1}-N_{2}\right)\left(K_{0}-K(X)\right),
$$

where $K_{0}=K(0)$ is the value of the Green's function $K(X)$ for the operator $\mathcal{Q}$ when the peakon positions coincide, so that $X=0$.

The second pair of equations in (11) may then be written as

$$
\begin{aligned}
\partial_{t} M_{1} & =-\partial_{s} N_{1}-\left(M_{1} M_{2}+\sigma N_{1} N_{2}\right) K^{\prime}(X), \\
\partial_{t} M_{2} & =-\partial_{s} N_{2}+\left(M_{1} M_{2}+\sigma N_{1} N_{2}\right) K^{\prime}(X), \\
\partial_{t} N_{1} & =\sigma \partial_{s} M_{1}+\left(N_{1} M_{2}-M_{1} N_{2}\right) \frac{K_{0}-K}{K_{0}+K} K^{\prime}(X), \\
\partial_{t} N_{2} & =\sigma \partial_{s} M_{2}+\left(N_{1} M_{2}-M_{1} N_{2}\right) \frac{K_{0}-K}{K_{0}+K} K^{\prime}(X) .
\end{aligned}
$$

Asymptotically, when the peakons are far apart, the system (13) simplifies, since $\frac{K_{0}-K}{K_{0}+K} \rightarrow 1$ and $K^{\prime}(X) \rightarrow 0$ as $|X| \rightarrow \infty$.

The system (13) has two immediate conservation laws obtained from their sums and differences,

$$
\partial_{t}\left(M_{1}+M_{2}\right)=-\partial_{s}\left(N_{1}+N_{2}\right), \quad \partial_{t}\left(N_{1}-N_{2}\right)=\sigma \partial_{s}\left(M_{1}-M_{2}\right) .
$$

These may be resolved by setting

$$
\begin{array}{ll}
M_{1}-M_{2}=\frac{\partial_{t} X}{K_{0}-K}, & N_{1}-N_{2}=\sigma \frac{\partial_{s} X}{K_{0}-K}, \\
M_{1}+M_{2}=\partial_{s} \phi, & N_{1}+N_{2}=-\partial_{t} \phi,
\end{array}
$$

and introducing two potential functions, $X$ and $\phi$, for which equality of cross derivatives will now produce the system of equations (12) and (13). Namely, from (14), one may solve for

$$
\begin{aligned}
M_{1} & =\frac{\partial_{t} X}{2\left(K_{0}-K\right)}+\frac{1}{2} \partial_{s} \phi, & N_{1} & =\frac{\sigma \partial_{s} X}{2\left(K_{0}-K\right)}-\frac{1}{2} \partial_{t} \phi, \\
M_{2} & =-\frac{\partial_{t} X}{2\left(K_{0}-K\right)}+\frac{1}{2} \partial_{s} \phi, & N_{2} & =-\frac{\sigma \partial_{s} X}{2\left(K_{0}-K\right)}-\frac{1}{2} \partial_{t} \phi .
\end{aligned}
$$

Now substituting into the first and third equations of the system (13) yields the determining equations for the potentials $X$ and $\phi$,

$$
\begin{aligned}
& \left(\partial_{t}^{2}+\sigma \partial_{s}^{2}\right) \phi+\frac{K^{\prime}}{K_{0}+K}\left(X_{t} \phi_{t}+\sigma X_{s} \phi_{s}\right)=0 \\
& \left(\partial_{t}^{2}+\sigma \partial_{s}^{2}\right) X+\frac{K^{\prime}}{2\left(K_{0}-K\right)}\left(X_{t}^{2}+\sigma X_{s}^{2}\right)=-\frac{1}{2} K^{\prime}\left(K_{0}-K\right)\left(\phi_{s}^{2}+\sigma \phi_{t}^{2}\right),
\end{aligned}
$$


A simplification. A simplification arises if $\phi=0$, in which case the collision is perfectly antisymmetric, as seen from equation (14). This is the peakon-antipeakon collision, for which the second equation in (16) reduces to

$$
\left(\partial_{t}^{2}+\sigma \partial_{s}^{2}\right) X+\frac{K^{\prime}}{2\left(K_{0}-K\right)}\left(X_{t}^{2}+\sigma X_{s}^{2}\right)=0
$$

This equation can be easily rearranged to produce a linear equation:

$$
\left(\partial_{t}^{2}+\sigma \partial_{s}^{2}\right) F(X)=0, \quad \text { where } \quad F(X)=\int_{X_{0}}^{X}\left(K_{0}-K(Y)\right)^{-1 / 2} d Y .
$$

Thus, the dynamics of the relative spacing $X(t, s)=Q^{1}-Q^{2}$ in the peakon-antipeakon collision may be obtained by elementary means. In particular, for the $H^{1}$ peakon case when $K(Y)=$ $\frac{1}{2} e^{-|Y|}$, we have

$$
F(X)=\sqrt{2} \int_{X_{0}}^{X} \frac{1}{\sqrt{1-e^{-|Y|}}} d Y .
$$

We can take for simplicity $X_{0}=0$, this would change $F(X)$ only by a constant. When $X>0$ the integral becomes

$$
\begin{aligned}
F(X) & =\sqrt{2} \int_{0}^{X} \frac{1}{\sqrt{1-e^{-Y}}} d Y=\sqrt{2} \int_{0}^{X} \frac{e^{Y / 2}}{\sqrt{e^{Y}-1}} d Y \\
& =2 \sqrt{2} \int_{0}^{X} \frac{d e^{Y / 2}}{\sqrt{\left(e^{Y / 2}\right)^{2}-1}}=2 \sqrt{2} \cosh ^{-1}\left(e^{X / 2}\right) .
\end{aligned}
$$

In general $F(X)=2 \sqrt{2} \operatorname{sign}(X) \cosh ^{-1}\left(e^{|X| / 2}\right)$. Hence the solution $X(t, s)$ can be expressed in terms of any given solution $h(t, s)$ of the linear equation $\left(\partial_{t}^{2}+\sigma \partial_{s}^{2}\right) h(t, s)=0$ as

$$
X(t, s)= \pm \ln \left(\cosh ^{2}(h(t, s))\right) .
$$

When $\sigma=1, h(t, s)$ is any harmonic function, when $\sigma=-1, h(t, s)$ is any solution of the wave equation.

\section{Complexified $G$-strand $\operatorname{Diff}(\mathbb{R})$ equations}

As mentioned in the Introduction, the class of problems being treated here generalizes the classical chiral model, which is a special case of the $G$-strands when their structure group is $\mathrm{SO}(3)$, as discussed in [14]. Harmonic maps are also related to the chiral model as discussed in [27], upon complexifying the independent coordinates of the chiral model. Pursuing this idea, we shall complexify the independent coordinates $(t, s)$ of the $G$-strand equations $(5)$ on the Lie group of diffeomorphisms $\operatorname{Diff}(\mathbb{R})$ and thereby derive a class of equations which may be regarded as partial differential equations for harmonic maps of $\mathbb{C}$ into $\operatorname{Diff}(\mathbb{R})$. This is potentially a rich area for further mathematical study.

\subsection{Complexifying coordinates $(t, s)$ for a real Lagrangian}

We complexify coordinates $(t, s) \in \mathbb{R}^{2} \rightarrow(z, \bar{z}) \in \mathbb{C}$, where $\bar{z}$ denotes the complex conjugate of $z$ and identify $v=\bar{u}$. Consequently, the Euler-Poincaré $G$-strand equations in (5) become

$$
\frac{\partial}{\partial z} \frac{\delta \ell}{\delta u}+\frac{\partial}{\partial \bar{z}} \frac{\delta \ell}{\delta \bar{u}}=-\operatorname{ad}_{u}^{*} \frac{\delta \ell}{\delta u}-\operatorname{ad}_{\bar{u}}^{*} \frac{\delta \ell}{\delta \bar{u}}, \quad \text { and } \quad \frac{\partial \bar{u}}{\partial z}-\frac{\partial u}{\partial \bar{z}}=\operatorname{ad}_{u} \bar{u}
$$


Here we assume that the Lagrangian $\ell$ is defined as

$$
\ell(u, \bar{u})=\frac{1}{2}\|u\|_{\mathcal{Q}}^{2}+\frac{\sigma}{2}\|\bar{u}\|_{\mathcal{Q}}^{2}
$$

where the operator $\mathcal{Q}$ and the expression $\|w\|_{\mathcal{Q}}$ are formally defined as before, although $\|w\|_{\mathcal{Q}}$ does not define a norm for complex $w$.

By evaluating $m=\delta \ell / \delta u=\mathcal{Q} u, \bar{m}=\delta \ell / \delta \bar{u}=\sigma \mathcal{Q} \bar{u}$, we observe that consistency of this construction requires that we set $\sigma=1$ in equation (19).

For the real Lagrangian $\ell$, equations (18) may be rewritten as

$$
\begin{aligned}
& m_{z}+\bar{m}_{\bar{z}}=-\operatorname{ad}_{u}^{*} m-\operatorname{ad}_{\bar{u}}^{*} \bar{m}=-(u m)_{x}-m u_{x}-(\bar{u} \bar{m})_{x}-\bar{m} \bar{u}_{x}, \\
& \bar{u}_{z}-u_{\bar{z}}=-\operatorname{ad}_{\bar{u}} u=-u \bar{u}_{x}+\bar{u} u_{x},
\end{aligned}
$$

where the independent coordinate $x \in \mathbb{R}$ is on the real line, although coordinates $(z, \bar{z}) \in \mathbb{C}$ are complex, as are the solutions $u$, and $m=\mathcal{Q} u$, of the equations (18) for the real Lagrangian (19) for $\sigma=1$.

\section{Remark 1.}

- Equations (20) are invariant under two involutions, $P$ and $C$, where

$$
P:(x, m) \rightarrow(-x,-m) \quad \text { and } \quad C: \text { complex conjugation. }
$$

- For real variables $m=\bar{m}, u=\bar{u}$ and real evolution parameter $z=\bar{z}=: t$, equations (20) reduce to the Camassa-Holm equation (7).

\subsection{Peakon solutions of complexified $\operatorname{Diff}(\mathbb{R})$-strand equations in (20)}

The complexified $\operatorname{Diff}(\mathbb{R})$-strand equations (20) admit peakon solutions in complex momenta $m$, with continuous complex velocity $u$. We state this result in the following theorem.

Theorem 3. The complexified $\operatorname{Diff}(\mathbb{R})$-strand equations (20) admit singular solutions expressed as linear superpositions summed over $a \in \mathbb{Z}$

$$
\begin{aligned}
& m(x, z, \bar{z})=\sum_{a} M_{a}(z, \bar{z}) \delta\left(x-Q^{a}(z, \bar{z})\right), \\
& u(x, z, \bar{z})=K * m=\sum_{a} M_{a}(z, \bar{z}) K\left(x, Q^{a}\right) .
\end{aligned}
$$

These are peakon solutions for $K(x, y)=\frac{1}{2} e^{-|x-y|}$. An important feature of these singular solutions is that $Q^{a}$ with $a \in \mathbb{Z}$ are real functions of $z$ and $\bar{z}$. These singular solutions follow from Hamilton's principle $\delta S=0$ for the constrained action $S=\int L(u, \bar{u}, Q) d t$ given by

$$
S=\int \ell(u, \bar{u})+\Re\left(\sum_{a} M_{a}(z, \bar{z})\left(\partial_{z} Q^{a}(z, \bar{z})-u\left(Q^{a}, z, \bar{z}\right)\right)\right) d z d \bar{z},
$$

where $\Re$ is the real part of the expression.

Proof. The proof follows the same lines as the proof in [14] for the real case, keeping in mind that the coordinate $x \in \mathbb{R}$ is a real independent variable.

The solution parameters $Q^{a}(z, \bar{z}) \in \mathbb{R}$ and $M_{a}(z, \bar{z}) \in \mathbb{C}$ with $a \in \mathbb{Z}$ that specify the singular solutions (21) are determined by the following set of evolutionary PDEs in $z$ and $\bar{z}$, in which we denote $K^{a b}:=K\left(Q^{a}, Q^{b}\right)$ with integer summation indices $b, c, e \in \mathbb{Z}$ :

$$
\partial_{z} Q^{a}(z, \bar{z})=u\left(Q^{a}, z, \bar{z}\right)=\sum_{b} M_{b}(z, \bar{z}) K^{a b}
$$




$$
\begin{aligned}
& \left.\partial_{z} M_{a}(z, \bar{z})=-\partial_{\bar{z}} \bar{M}_{a}-\sum_{c}\left(M_{a} M_{c}+\bar{M}_{a} \bar{M}_{c}\right) \frac{\partial K^{a c}}{\partial Q^{a}} \quad \text { (no sum on } a\right), \\
& \partial_{z} \bar{M}_{a}(z, \bar{z})=\partial_{\bar{z}} M_{a}+\sum_{b, c, e}\left(\bar{M}_{b} M_{c}-M_{b} \bar{M}_{c}\right) \frac{\partial K^{e c}}{\partial Q^{e}}\left(K^{e b}-K^{c b}\right)\left(K^{-1}\right)_{a e}
\end{aligned}
$$

The last pair of equations in (22) may be solved as a system for the momenta, or Lagrange multipliers $\left(M_{a}\right)$, then used in the first equation to update the support set of positions $Q^{a}(z, \bar{z})$. Given $Q^{a}(z, \bar{z})$ for $a \in \mathbb{Z}$, one constructs $m(x, z, \bar{z})$ along the solution paths $x=Q^{a}(z, \bar{z})$ from the first equation of $(21)$ and then obtains $u(x, z, \bar{z})$ for $x \in \mathbb{R}$ from the second equation of (21). Alternatively, knowing the position $Q^{a}(z, \bar{z}), a \in \mathbb{Z}$, for all $z$ and $\bar{z}$, one also determines $\bar{M}_{a}$ upon inverting the matrix $K^{a b}$ in the third equation in (22). Note that the third equation in (22) is not the complex conjugate of the previous one.

Single-peakon solution. The single-peakon solution is again straightforward to obtain from equations $(22)$. The solution $Q^{1}(z, \bar{z})$ satisfies the (linear) two-dimensional wave equation.

Two-peakon solution. The two-peakon collision for the complexified $G$-strand equation emerges by following the same path as in the previous section 2.3 with appropriate minor changes. In particular, equation (15) becomes

$$
M_{1}=\frac{\partial_{z} X}{2\left(K_{0}-K\right)}+\frac{i}{2} \partial_{\bar{z}} \phi, \quad M_{2}=-\frac{\partial_{z} X}{2\left(K_{0}-K\right)}+\frac{i}{2} \partial_{\bar{z}} \phi
$$

where $\phi(z, \bar{z})$ is real. Now substituting into the second and third equations of the system (22) we obtain

$$
\begin{aligned}
& \phi_{z z}+\phi_{\bar{z} \bar{z}}+\frac{K^{\prime}}{K_{0}+K}\left(X_{z} \phi_{z}+X_{\bar{z}} \phi_{\bar{z}}\right)=0 \\
& X_{z z}+X_{\bar{z} \bar{z}}+\frac{K^{\prime}}{2\left(K_{0}-K\right)}\left(X_{z}^{2}+X_{\bar{z}}^{2}\right)=\frac{1}{2} K^{\prime}\left(K_{0}-K\right)\left(\phi_{z}^{2}+\phi_{\bar{z}}^{2}\right) .
\end{aligned}
$$

Introducing the notation

$$
\partial_{z}=\partial_{\xi}+i \partial_{\eta} \quad \text { and } \quad \partial_{\bar{z}}=\partial_{\xi}-i \partial_{\eta}
$$

we obtain the following PDE system for the real variables $\xi, \eta$ :

$$
\begin{aligned}
\phi_{\xi \xi}-\phi_{\eta \eta}+\frac{K^{\prime}}{K_{0}+K}\left(X_{\xi} \phi_{\xi}-X_{\eta} \phi_{\eta}\right) & =0 \\
X_{\xi \xi}-X_{\eta \eta}+\frac{K^{\prime}}{2\left(K_{0}-K\right)}\left(X_{\xi}^{2}-X_{\eta}^{2}\right) & =\frac{1}{2} K^{\prime}\left(K_{0}-K\right)\left(\phi_{\xi}^{2}-\phi_{\eta}^{2}\right) .
\end{aligned}
$$

When $\phi=0$, we again have peakon-antipeakon collisions with a simple solution as in Section 2.3. The equation reduces to the two-dimensional (linear) wave equation:

$$
\left(\partial_{\xi}^{2}-\partial_{\eta}^{2}\right) F(X)=0
$$

where $F$ is the same function as in (17). The obvious difference when $\sigma=1$ is that the Laplacian $\partial_{s}^{2}+\partial_{t}^{2}$ in (16) for real parameters $(t, s)$ is replaced in (23) by the wave operator $\partial_{\xi}^{2}-\partial_{\eta}^{2}$ for this complex case. 


\subsection{Complexified $\operatorname{Diff}(\mathbb{R})$-strand equations for a real Lagrangian given by a complex norm}

Here the Lagrangian $\ell$ is taken to be real, and defined by a complex norm as

$$
\ell(u, \bar{u})=\int u \mathcal{Q} \bar{u} d x
$$

where the operator $\mathcal{Q}$ is defined as before - a real, symmetric, positive-definite operator, invariant under spatial translations in $x \in \mathbb{R}$, and with vanishing boundary conditions as $|x| \rightarrow \infty$.

Since the Lagrangian $\ell$ is chosen to be a norm on complex functions defined by the spatial differential operator $\mathcal{Q}$, we may regard the first equation in the complexified $G$-strand system (18) as describing geodesic motion according to the map $(z, \bar{z}) \in \mathbb{C} \rightarrow \operatorname{Diff}(\mathbb{R})$ with respect to the above norm. The second equation (18) may be regarded as a compatibility (or zero-curvature) condition in the complex parameters $z$ and $\bar{z}$.

Evaluating $\delta \ell / \delta u=\mathcal{Q} \bar{u}, \delta \ell / \delta \bar{u}=\mathcal{Q} u$, the equations (18) for the real Lagrangian (24) may be rewritten as

$$
\begin{aligned}
\bar{\mu}_{z}+\mu_{\bar{z}} & =-\operatorname{ad}_{u}^{*} \bar{\mu}-\operatorname{ad}_{\bar{u}}^{*} \mu=-(u \bar{\mu})_{x}-\bar{\mu} u_{x}-(\bar{u} \mu)_{x}-\mu \bar{u}_{x}, \\
\bar{u}_{z}-u_{\bar{z}} & =-\operatorname{ad}_{\bar{u}} u=-u \bar{u}_{x}+\bar{u} u_{x},
\end{aligned}
$$

where $\mu=\mathcal{Q} u$ for convenience.

\section{Remark 2.}

- Equations (25) are invariant under the involutions, $P$ and $C$, where

$$
P:(x, \mu) \rightarrow(-x,-\mu) \text { and } C: \text { complex conjugation. }
$$

- For real variables $\mu=\bar{\mu}:=m, u=\bar{u}$ and real evolution parameter $z=\bar{z}=: t$, equations (25) reduce to the Camassa-Holm equation (7).

Similarly to the previous case, the singular solutions expressed as linear superpositions summed over $a \in \mathbb{Z}$

$$
\begin{aligned}
& \mu(x, z, \bar{z})=\sum_{a} M_{a}(z, \bar{z}) \delta\left(x-Q^{a}(z, \bar{z})\right), \\
& u(x, z, \bar{z})=K * \mu=\sum_{a} M_{a}(z, \bar{z}) K\left(x, Q^{a}\right) .
\end{aligned}
$$

The corresponding equations for the solution parameters $Q^{a}(z, \bar{z}) \in \mathbb{R}$ and $M_{a}(z, \bar{z}) \in \mathbb{C}$ with $a \in \mathbb{Z}$ are

$$
\begin{aligned}
& \partial_{z} Q^{a}(z, \bar{z})=u\left(Q^{a}, z, \bar{z}\right)=\sum_{b} M_{b}(z, \bar{z}) K^{a b} \\
& \left.\partial_{z} \bar{M}_{a}(z, \bar{z})=-\partial_{\bar{z}} M_{a}-\sum_{c}\left(\bar{M}_{a} M_{c}+M_{a} \bar{M}_{c}\right) \frac{\partial K^{a c}}{\partial Q^{a}} \quad \text { (no sum on } a\right), \\
& \partial_{z} \bar{M}_{a}(z, \bar{z})=\partial_{\bar{z}} M_{a}+\sum_{b, c, e}\left(\bar{M}_{b} M_{c}-M_{b} \bar{M}_{c}\right) \frac{\partial K^{e c}}{\partial Q^{e}}\left(K^{e b}-K^{c b}\right)\left(K^{-1}\right)_{a e} .
\end{aligned}
$$

The difference with the previous case is only in the second equation, since the compatibility equations are the same. 
One-peakon solution. For the one-peakon solution $\partial_{\bar{z}} M_{1}=0$, and therefore $\partial_{z} \partial_{\bar{z}} Q^{1}=0$. Thus $Q^{1}$ satisfies the Laplace equation $\Delta Q^{1}=0$, and the solution is given by any harmonic function.

Two-peakon solution. The two-peakon collision for this complexified $G$-strand equation can be obtained in a similar fashion. This time the expressions are

$$
M_{1}=\frac{\partial_{z} X}{2\left(K_{0}-K\right)}+\frac{i}{2} \partial_{z} \phi, \quad M_{2}=-\frac{\partial_{z} X}{2\left(K_{0}-K\right)}+\frac{i}{2} \partial_{z} \phi,
$$

where $\phi(z, \bar{z})$ is real. Now substituting into the second and third equations of the system (26) we obtain

$$
\begin{aligned}
& \phi_{z \bar{z}}+\frac{K^{\prime}}{2\left(K_{0}+K\right)}\left(X_{z} \phi_{\bar{z}}+X_{\bar{z}} \phi_{z}\right)=0 \\
& X_{z \bar{z}}+\frac{K^{\prime}}{2\left(K_{0}-K\right)} X_{z} X_{\bar{z}}=-\frac{1}{2} K^{\prime}\left(K_{0}-K\right) \phi_{z} \phi_{\bar{z}} .
\end{aligned}
$$

With the notations $\partial_{z}=\partial_{\xi}+i \partial_{\eta}$ and $\partial_{\bar{z}}=\partial_{\xi}-i \partial_{\eta}$ we obtain the following system for the real variables $\xi, \eta$ :

$$
\begin{aligned}
& \phi_{\xi \xi}+\phi_{\eta \eta}+\frac{K^{\prime}}{K_{0}+K}\left(X_{\xi} \phi_{\xi}+X_{\eta} \phi_{\eta}\right)=0 \\
& X_{\xi \xi}+X_{\eta \eta}+\frac{K^{\prime}}{2\left(K_{0}-K\right)}\left(X_{\xi}^{2}+X_{\eta}^{2}\right)=-\frac{1}{2} K^{\prime}\left(K_{0}-K\right)\left(\phi_{\xi}^{2}+\phi_{\eta}^{2}\right) .
\end{aligned}
$$

When $\phi=0$, we again have peakon-antipeakon collisions with a simple solution as in Section 2.3. The equation reduces to the Laplace equation:

$$
\left(\partial_{\xi}^{2}+\partial_{\eta}^{2}\right) F(X)=0
$$

where again $F$ is the same as in (17).

The role of the Laplacian has been studied before in connection with the relation between chiral models and harmonic maps in [27]. See [9] for further discussion and a guide to the modern literature. The corresponding studies for $G$-strands can be expected to be developed further in future work. For example, it may be interesting to study the extension of $G$-strand equations to supersymmetric variables following methods considered in [10, 24], particularly for $G=\operatorname{Diff}(\mathbb{R})$, if the singular solutions would be preserved.

\section{Conclusions}

This paper has used the Euler-Poincaré (EP) framework for studying the $G$-strand equations, comprising the system of partial differential equations obtained from the EP variational equations (5) for a $G$-invariant Lagrangian, coupled to an auxiliary zero-curvature equation. The latter has often been the departure point and main focus in other approaches, especially for the integrable chiral models, where it sets up the Lax-pair formulation of the system [18].

Once the $G$-invariant Lagrangian has been specified, the combined dynamics and zerocurvature system of $G$-strand equations in (5) follows automatically in the EP framework.

The single-peakon solution of the $\operatorname{Diff}(\mathbb{R})$-strand equations (5) has been reduced to a solution of the linear Laplace or wave equations. The stability of the single-peakon solution under perturbations into the full solution space of equations (5) would be an interesting problem for future work.

The pairwise dynamics of the singular peakon solutions for the $G$-strand equations with $G=$ $\operatorname{Diff}(\mathbb{R})$ has also been studied. The peakon-peakon and peakon-antipeakon collision interactions 
have been found to admit elementary solution methods which reduce again to solving the linear Laplace or wave equations.

Finally, two complexified version of the $\operatorname{Diff}(\mathbb{R})$-strand equations has been introduced and their peakon collision solutions have been formulated and again solved by elementary means. In the latter case, the analogous peakon-peakon and peakon-antipeakon collision interactions involve the solution of the Laplace or wave operator in each case.

\section{Acknowledgements}

We thank our friends A.M. Bloch, C.J. Cotter, F. Gay-Balmaz, A. Iserles, J.R. Percival, T.S. Ratiu and C. Tronci for their kind encouragement and thoughtful remarks during the course of this work. We are thankful also to Dr. Sergey Kushnarev and an anonymous referee whose comments and suggestions have helped us a lot in the revision of this paper. DDH gratefully acknowledges partial support by the Royal Society of London's Wolfson Award scheme

and the European Research Council's Advanced Grant 267382 FCCA. RII is supported by the Science Foundation Ireland (SFI), under Grant No. 09/RFP/MTH2144.

\section{References}

[1] Camassa R., Holm D.D., An integrable shallow water equation with peaked solitons, Phys. Rev. Lett. 71 (1993), 1661-1664, patt-sol/9305002.

[2] Cherednik I.V., On the integrability of the 2-dimensional asymmetric chiral $\mathrm{O}(3)$ field equations and their quantum analogue, Soviet J. Nuclear Phys. 33 (1981), 144-145.

[3] De Vega H.J., Field theories with an infinite number of conservation laws and Bäcklund transformations in two dimensions, Phys. Lett. B 87 (1979), 233-236.

[4] Ellis D.C.P., Gay-Balmaz F., Holm D.D., Putkaradze V., Ratiu T.S., Symmetry reduced dynamics of charged molecular strands, Arch. Ration. Mech. Anal. 197 (2010), 811-902, arXiv:0901.2959.

[5] Gay-Balmaz F., Clebsch variational principles in field theories and singular solutions of covariant EPDiff equations, Rep. Math. Phys. 71 (2013), 231-277, arXiv:1209.0109.

[6] Gay-Balmaz F., Ratiu T.S., The geometric structure of complex fluids, Adv. in Appl. Math. 42 (2009), 176-275, arXiv:0903.4294.

[7] Gibbons J., Holm D.D., Kupershmidt B., Gauge-invariant Poisson brackets for chromohydrodynamics, Phys. Lett. A 90 (1982), 281-283.

[8] Gibbons J., Holm D.D., Kupershmidt B., The Hamiltonian structure of classical chromohydrodynamics, Phys. D 6 (1983), 179-194.

[9] Guest M.A., Harmonic maps, loop groups, and integrable systems, London Mathematical Society Student Texts, Vol. 38, Cambridge University Press, Cambridge, 1997.

[10] Guha P., Olver P.J., Geodesic flow and two (super) component analog of the Camassa-Holm equation, SIGMA 2 (2006), 054, 9 pages, nlin.SI/0605041.

[11] Hlavatý L., Šnobl L., Principal chiral models on non-semisimple groups, J. Phys. A: Math. Gen. 34 (2001), 7795-7809.

[12] Holm D.D., Euler-Poincaré dynamics of perfect complex fluids, in Geometry, Mechanics, and Dynamics, Editors P. Newton, P. Holmes, A. Weinstein, Springer, New York, 2002, 113-167, nlin.CD/0103041.

[13] Holm D.D., Geometric mechanics. Part II. Rotating, translating and rolling, 2nd ed., Imperial College Press, London, 2011.

[14] Holm D.D., Ivanov R.I., Percival J.R., G-strands, J. Nonlinear Sci. 22 (2012), 517-551, arXiv:1109.4421.

[15] Holm D.D., Kupershmidt B.A., The analogy between spin glasses and Yang-Mills fluids, J. Math. Phys. 29 (1988), 21-30.

[16] Holm D.D., Marsden J.E., Momentum maps and measure-valued solutions (peakons, filaments, and sheets) for the EPDiff equation, in The Breadth of Symplectic and Poisson Geometry, Progr. Math., Vol. 232, Editors J.E. Marsden, T.S. Ratiu, Birkhäuser Boston, Boston, MA, 2005, 203-235, nlin.CD/0312048. 
[17] Holm D.D., Marsden J.E., Ratiu T.S., The Euler-Poincaré equations and semidirect products with applications to continuum theories, Adv. Math. 137 (1998), 1-81, chao-dyn/9801015.

[18] Lax P.D., Integrals of nonlinear equations of evolution and solitary waves, Comm. Pure Appl. Math. 21 (1968), 467-490.

[19] Maillet J.-M., Hamiltonian structures for integrable classical theories from graded Kac-Moody algebras, Phys. Lett. B 167 (1986), 401-405.

[20] Mañas M., The principal chiral model as an integrable system, in Harmonic Maps and Integrable Systems, Aspects Math., Vol. E23, Vieweg, Braunschweig, 1994, 147-173.

[21] Marsden J.E., Ratiu T.S., Introduction to mechanics and symmetry. A basic exposition of classical mechanical systems, Texts in Applied Mathematics, Vol. 17, Springer-Verlag, New York, 1994.

[22] Novikov S., Manakov S.V., Pitaevskiǔ L.P., Zakharov V.E., Theory of solitons. The inverse scattering method, Contemporary Soviet Mathematics, Plenum, New York, 1984.

[23] Pohlmeyer K., Integrable Hamiltonian systems and interactions through quadratic constraints, Comm. Math. Phys. 46 (1976), 207-221.

[24] Popowicz Z., A 2-component or $N=2$ supersymmetric Camassa-Holm equation, Phys. Lett. A 354 (2006), 110-114, nlin.SI/0509050.

[25] Sochen N., Integrable generalized principal chiral models, Phys. Lett. B 391 (1997), 374-380, hep-th/9607009.

[26] Tronci C., Hybrid models for complex fluids with multipolar interactions, J. Geom. Mech. 4 (2012), 333-363, arXiv:1011.4389.

[27] Uhlenbeck K., Harmonic maps into Lie groups: classical solutions of the chiral model, J. Differential Geom. 30 (1989), 1-50.

[28] Ward R.S., Soliton solutions in an integrable chiral model in $2+1$ dimensions, J. Math. Phys. 29 (1988), 386-389.

[29] Witten E., Non-abelian bosonization in two dimensions, Comm. Math. Phys. 92 (1984), 455-472.

[30] Yanovski A.B., Bi-Hamiltonian formulation of the $\mathrm{O}(3)$ chiral fields equations hierarchy via a polynomial bundle, J. Phys. A: Math. Gen. 31 (1998), 8709-8726.

[31] Zakharov V.E., Mikhailov A.V., On the integrability of classical spinor models in two-dimensional spacetime, Comm. Math. Phys. 74 (1980), 21-40.

[32] Zakharov V.E., Mikhailov A.V., Relativistically invariant two-dimensional models of field theory which are integrable by means of the inverse scattering problem method, Soviet Phys. JETP 47 (1978), 1017-1027. 